\title{
A SINGULAR INTEGRAL INEQUALITY ON A BOUNDED INTERVAL
}

\author{
J. S. BRADLEY ${ }^{1}$ AND W. N. EVERITT
}

\begin{abstract}
An inequality of the form (1.1) is established, where $p, q$ are real-valued functions on an interval $[a, b)$ of the real line, with $-\infty<a<b$ $<\infty, p(x)>0$ on $[a, b), \mu_{0}$ is a real number and $f$ is a complex-valued function in a linear manifold so chosen that all three integrals in (1.1) are absolutely convergent. The problem is singular in that while $p^{-1} \in L(a, b)$ we require $q$ to have a behavior at $b$ of such a form that $q \notin L(a, b)$.
\end{abstract}

1. Introduction. An inequality of the form

$$
\int_{a}^{b}\left[p\left|f^{\prime}\right|^{2}+q|f|^{2}\right] \geqslant \mu_{0} \int_{a}^{b}|f|^{2} \quad(f \in D)
$$

is established, where $p, q$ are real-valued functions on an interval $[a, b)$ of the real line, with $-\infty<a<b<\infty, p(x)>0$ on $[a, b)$, $\mu_{0}$ is a real number and $f$ is a complex-valued function in a linear manifold so chosen that all three integrals in (1.1) are absolutely convergent. The problem is singular in that while $p^{-1} \in L(a, b)$ we require $q$ to have a behavior at $b$ of such a form that $q \notin L(a, b)$.

We have established, in a previous paper [1], an inequality of the form (1.1) for the regular case, i.e., $p^{-1}$ and $q$ integrable on $[a, b]$, and also for the singular case where $b=\infty$. Some recent work by Everitt and Giertz [4] and by Kalf [5] make it feasible to study singular inequalities of the form (1.1) on bounded half-open intervals.

The Euler equation for minimizing the left-hand side of (1.1) is

$$
M[y]=\lambda y \quad \text { on }[a, b)
$$

where $\lambda$ is a parameter and $M[y]$ is the second-order linear differential expression

$$
M[y]=-\left(p y^{\prime}\right)^{\prime}+q y \quad \text { on }[a, b) \quad\left({ }^{\prime} \equiv d / d x\right)
$$

Presented to the Society, January 23, 1975 under the title Singular integro-differential inequalities; received by the editors March 24, 1975 and, in revised form, July 29, 1975.

AMS (MOS) subject classifications (1970). Primary 26A84, 34B25; Secondary 47B10, 47E05.

1 This author gratefully acknowledges a travel grant from the British Science Research Council that enabled him to visit the University of Dundee in the Summer of 1974 where this work was begun.

Copyright $\odot 1977$, American Mathematical Society 
We make use of certain well-known relationships between equation (1.2) and inequality (1.1) from the calculus of variations, but we do not require that the functions in $D$ for which (1.1) holds satisfy specific boundary conditions at the endpoints $a$ and $b$ as is common when such problems are considered by methods in the calculus of variations.

We use the following notations: $L(a, b)$ and $L^{2}(a, b)$ denote the classical Lebesgue complex integration spaces; a property is 'loc' on $[a, b)$ if it is satisfied on all compact subintervals of $[a, b)$; AC represents absolute continuity. Thus $\mathrm{AC}_{\text {loc }}[a, b)$ is the class of all functions that are absolutely continuous on compact subintervals of $[a, b)$.

The coefficient functions $p$ and $q$ are required to satisfy the following basic conditions:

(i) $p \in \mathrm{AC}_{\mathrm{loc}}[a, b), p(x)>0,(x \in[a, b))$;

(ii) both $p^{\prime}$ and $q$ belong to $L_{\mathrm{loc}}^{2}[a, b)$.

Note that these conditions imply that the differential expression $M$ is regular at all points of $[a, b)$. (See $[6, \S 16.1]$.) We remark below on the need for (ii).

Following the notation in $[1, \S 2]$ we define the following sets of functions:

(i) $\Delta=\left\{f \in L^{2}(a, b): f^{\prime} \in \operatorname{AC}_{\mathrm{loc}}[a, b), M[f] \in L^{2}(a, b)\right\}$;

(ii) for $\alpha \in[0, \pi), \mathscr{D}(\alpha)=\left\{f \in \Delta: f(a) \cos \alpha+f^{\prime}(a) \sin \alpha=0\right\}$;

(iii) $D=\left\{f \in L^{2}(a, b): f \in \operatorname{AC}_{\mathrm{loc}}[a, b), p^{1 / 2} f^{\prime},|q|^{1 / 2} f \in L^{2}(a, b)\right\}$.

It should be noted that (ii)(1.3) implies that $\Delta$ contains all infinitely differentiable functions with compact support in $(a, b)$ and so $\Delta$ is dense in $L^{2}(a, b)$.

For each $\alpha \in[0, \pi)$, an operator $T(\alpha)$ is defined by

$$
\text { domain of } T(\alpha) \text { is } \mathscr{D}(\alpha) \text { and } T(\alpha) f=M[f] \text {. }
$$

It is known that $T(\alpha)$ is selfadjoint in $L^{2}(a, b)$ if, and only if, $M$ is limit-point at the singular endpoint $b$. (See $[6, \S 18.3]$.)

Additionally we assume the following conditions on the coefficient functions $p$ and $q$ :

(i) $p^{-1} \in L(a, b)$;

(ii) $\int_{a}^{b} q_{+}=\infty$, where $q_{+}=(q+|q|) / 2$.

Both conditions are needed in the proof of our theorem. The second condition (ii) insures that $q \notin L(a, b)$ and so forces $b$ to be a singular endpoint for the differential expression $M[6, \S 16.1]$. This then is a distinct departure from the work contained in $[1, \S 2]$.

Finally, the following conditions are required: 
(i) $M$ satisfies the Dirichlet condition at $b$, i.e., $p^{1 / 2} f^{\prime}$ and $|q|^{1 / 2} f \in L^{2}(a, b)$ for all $f \in \Delta ;$

(ii) the operator $T\left(\frac{1}{2} \pi\right)$ is bounded below in $L^{2}(a, b)$;

i.e., there is a real number $\mu_{0}$ such that

$$
\left(T\left(\frac{1}{2} \mu\right) f, f\right) \geqslant \mu_{0}(f, f) \text { for all } f \in \mathscr{D}\left(\frac{1}{2} \pi\right),
$$

where $(\cdot, \cdot)$ is the usual inner product in $L^{2}(a, b)$.

To be exact we define $\mu_{0}=\inf \left\{\lambda: \lambda\right.$ is in the spectrum of $\left.T\left(\frac{1}{2} \pi\right)\right\}$, so that condition (ii) of (1.6) is equivalent to the assumption that $\mu_{0}>-\infty$.

Specific conditions on the coefficients $p$ and $q$ to insure that $M$ and $T\left(\frac{1}{2} \pi\right)$ satisfy (i) and (ii) of (1.6) may be found in the papers of Everitt and Giertz [4] and Kalf [5]. The results of these papers make it reasonable to assume (1.6) as a set of conditions to be satisfied and so indirectly impose conditions on the coefficients $p$ and $q$.

In [4] it is assumed that $p=1$ on $[a, b)$ and that $q$ satisfies a growth condition near $b$ which insures (ii) of (1.5), (i) and (ii) of (1.6) are satisfied. In [5] a general condition is given that insures that (1.6) is satisfied, but it is then necessary to require $q$ to satisfy (ii) of (1.5).

In [5] Kalf has shown that conditions (ii) of (1.5) and (i) of (1.6) imply that $M$ is strong limit-point at the singular endpoint $b$, i.e.,

$$
\lim _{x \rightarrow b^{-}} p(x) f(x) g^{\prime}(x)=0 \quad(f, g \in \Delta) .
$$

An alternative proof of this result may be found in the paper by Everitt [3]. Note that (1.7) implies that $M$ is limit-point at $b$ and so all the operators $T(\alpha)(\alpha \in[0, \pi))$ are selfadjoint in $L^{2}(a, b)$.

We can now state the main result of this paper, which is,

THEOREM 1. If $p$ and $q$ are real-valued functions for which conditions (1.5) and (1.6) hold, then inequality (1.1) is valid for all functions $f$ in the set $D$ described in (1.4)(iii), with $\mu_{0}$ the smallest number in the spectrum of the operator $T\left(\frac{1}{2} \pi\right)$.

If $\mu_{0}$ is in the point or point-continuous spectrum of $T\left(\frac{1}{2} \pi\right)$, then there is equality in (1.1) if, and only if, $f=c \psi_{0}$ where $c$ is a complex number and $\psi_{0}$ is an eigenfunction for $T\left(\frac{1}{2} \pi\right)$ corresponding to $\mu_{0}$.

If $\mu_{0}$ is in the continuous spectrum of $T\left(\frac{1}{2} \pi\right)$, then there is equality in (1.1) if, and only if, $f$ is the zero function. The inequality is the best possible in the sense that there is a sequence $\left\{f_{n}\right\}$ such that $f_{n} \in D, \int_{a}^{b}\left|f_{n}\right|^{2}=1(n=1,2, \ldots)$ and

$$
\lim _{n \rightarrow \infty} \int_{a}^{b}\left[p\left|f_{n}^{\prime}\right|^{2}+q\left|f_{n}\right|^{2}\right]=\mu_{0}
$$

Our proof of Theorem 1 depends upon the following approximation theorem.

THEOREM 2. If the hypothesis of Theorem 1 holds, then for each $\varepsilon>0$ and each real-valued function $f$ in $D$ there is a real-valued function $g$ in $D\left(\frac{1}{2} \pi\right)$ such that 
$\left|\int_{a}^{b} p f^{\prime 2}-\int_{a}^{b} p g^{\prime 2}\right|<\varepsilon, \quad\left|\int_{a}^{b} f^{2}-\int_{a}^{b} g^{2}\right|<\varepsilon, \quad\left|\int_{a}^{b} q f^{2}-\int_{a}^{b} q g^{2}\right|<\varepsilon$.

The proofs of these theorems are in the next section.

2. Proofs. We restrict our attention in this section to real-valued functions since it is sufficient to prove (1.1) for all real-valued functions in the set $D$; this is a consequence of taking the coefficients $p$ and $q$ to be real-valued on $[a, b)$.

We use the following lemma in our proof of Theorem 2 ; see also $[1, \S 3]$.

LEMMA. If the hypothesis of Theorem 1 holds, $f \in D$ and $\varepsilon_{1}>0$, then there is a number $X$ in $(a, b)$ with the property that

$$
\int_{X}^{b} p f^{\prime 2}<\varepsilon_{1}, \quad \int_{X}^{b}|q| f^{2}<\varepsilon_{1}, \quad \int_{X}^{b} f^{2}<\varepsilon_{1}
$$

and

$$
f^{2}(X) \int_{a}^{X}|q|<\varepsilon_{1}, \quad(X-a) f^{2}(X)<\varepsilon_{1} .
$$

Proof. The fact that (2.1) holds follows from the fact that $f \in D$. To obtain (2.2) we first observe that $f$ in $D$ and (1.6)(i) imply that $\lim _{x \rightarrow b^{-}} f(x)$ exists. Indeed,

$$
f(x)=f(a)+\int_{a}^{x} f^{\prime}=f(a)+\int_{a}^{x} p^{-1 / 2} p^{1 / 2} f^{\prime}
$$

the last integral converges as $x \rightarrow b^{-}$since $p^{-1 / 2}$ and $p^{1 / 2} f^{\prime} \in L^{2}(a, b)$.

If $\lim _{x \rightarrow b^{-}} f(x) \neq 0$, then there exist numbers $k>0$ and $t \in[a, b)$ such that $f^{2}(x) \geqslant k$ for $x \in(t, b)$. Then

$$
\int_{a}^{x} q^{+} f^{2} \geqslant \int_{a}^{t} q^{+} f^{2}+k \int_{t}^{x} q^{+} .
$$

This inequality and (1.5)(ii) imply that $\int_{a}^{b} q^{+} f^{2}=\infty$ which is contrary to the assumption that $|q|^{1 / 2} f \in L^{2}$; i.e., that $f \in D$. Therefore $\lim _{x \rightarrow b^{-}} f(x)=0$.

It now follows that $(x-a) f^{2}(x) \rightarrow 0$ as $x \rightarrow b$. To complete the proof of the lemma it is sufficient to show that there is a sequence $\left\{x_{n}\right\}, x_{n} \rightarrow b$, for which $f^{2}\left(x_{n}\right) \int_{a}^{x_{n}}|q| \rightarrow 0$ as $n \rightarrow \infty$. If no such sequence exists, then there is a $d>0$ and $t \in(a, b)$ such that

$$
f^{2}(x) \geqslant d / \int_{a}^{x}|q| \quad(x \in(t, b)) .
$$

Multiplying by $|q|$ and integrating from $t$, we obtain

$$
\int_{t}^{x}|q| f^{2} \geqslant d\left[\log \int_{a}^{x}|q|-\log \int_{a}^{t}|q|\right] .
$$

This inequality, (1.5)(ii) and (1.6)(i) are incompatible with $f \in D$.

Proof of Theorem 2. For a positive number $\varepsilon_{1}$ we choose $X$ in $(a, b)$ so that the conclusion of the lemma is valid. Then we note that $f \in D$ implies that 
$f^{\prime} \in L^{2}(a, X)$ and that the set of continuously differentiable functions vanishing together with their derivativès at $a$ and $X$ is dense in $L^{2}(a, X)$. Therefore, for $\eta>0$, we may choose a continuously differentiable function $\phi$ such that $\phi(a)=\phi^{\prime}(a)=\phi(X)=\phi^{\prime}(X)=0, \phi(x)=0$ on $[X, b)$ and $\int_{a}^{X}\left|f^{\prime}-\phi\right|^{2}<\eta$. The function $g$ is defined by

$$
\begin{array}{ll}
g(x)=-\int_{x}^{X} \phi & (x \in[a, X]), \\
g(x)=0 & (x \in(X, b)) .
\end{array}
$$

It is clear that $g \in \mathcal{D}\left(\frac{1}{2} \pi\right)$ since $g^{\prime}(a)=0, g$ has a continuous second derivative, and $p, p^{\prime}$ and $q$ are all in $L^{2}(a, X)$, in view of the conditions (1.3). We note here that conditions (1.3) are essential to our argument, as were similar conditions in $[1, \S 3]$, and that it remains undecided whether condition (ii) of (1.3) could be replaced by the weaker assumption that $p^{\prime}$ and $q$ are locally integrable.

Using the function $g$ defined above and the lemma of this section, the estimates obtained in $[1, \S 3]$ remain valid, with $\infty$ replaced by $b$, and the proof of Theorem 2 proceeds in the same way as the proof of Theorem 3 of [1].

Proof of TheOrem 1. The proof of Theorem 1 is accomplished in three stages. The first is to establish inequality (1.1) for functions $f$ in $D\left(\frac{1}{2} \pi\right)$, the second is to extend the validity of the inequality to all of $D$, and the third is to determine the cases of equality. Each of these steps is similar to a corresponding part of the proof of Theorem 2 of [1] and we therefore limit our discussion here to a statement of the basic ideas involved and points where something needs to be added to the previous argument.

We begin by showing that (1.1) holds for $f$ in $\mathscr{D}\left(\frac{1}{2} \pi\right)$. Indeed, for such an $f$ an integration by parts, an application of (1.7) and condition (1.6) yield

$$
\int_{a}^{b}\left[p f^{\prime 2}+q f^{2}\right]=\int_{a}^{b} f M[f] \geqslant \mu_{0} \int_{a}^{b} f^{2},
$$

which establishes (1.1) for $f$ in $\mathscr{D}\left(\frac{1}{2} \pi\right)$. Since this argument appears to be identical to the one used in [1] it should be pointed out that an analogue of (1.7) is used in [1] and the arguments used in proving this analogue cannot be used in proving (1.7).

To prove that (1.1) holds for functions in $D$, we assume there is a function in $D$ for which (1.1) fails to hold and use the results of Theorem 2 to obtain a contradiction of the fact that (1.1) holds for all functions in $\mathscr{D}\left(\frac{1}{2} \pi\right)$. The details are the same as those used in [1].

The third stage of the proof, that of determining the cases of equality, separates into two cases according as $\mu_{0}$ is an eigenvalue or not.

If $\mu_{0}$ is in the point spectrum or point-continuous spectrum, then it is clear that there is equality in (1.1) for any constant multiple of an eigenfunction corresponding to $\mu_{0}$. Conversely, if equality holds in (1.1) for some $f$ in $D$, then it follows from Theorem 4 of [1] that $f$ is a solution of the differential equation $M[f]=\mu_{0} f$, and since the differential expression $M$ is in the limit point case at $b$ it follows that $f=c \psi_{0}$, where $\psi_{0}$ is an eigenfunction corresponding to $\mu_{0}$ and $c$ is a constant. 
If $\mu_{0}$ is in the continuous spectrum, we assume there is a function $f$ in $D$, $f \not \equiv 0$ for which equality holds. Again using Theorem 4 of [1] we find that $f$ is a solution of equation (1.2) for $\lambda=\mu_{0}$ and apply (1.7), Dirichlet condition (1.6)(i) and an integration by parts to conclude that $f(a) f^{\prime}(a)=0$. Since $\mu_{0}$ is not an eigenvalue for $T\left(\frac{1}{2} \pi\right), f^{\prime}(a) \neq 0$; therefore, $f(a)=0$ and $\mu_{0}$ is an eigenvalue of the operator $T(0)$. That this is impossible is established as in [1] using analytic properties of the Weyl $m$-coefficients $m(\lambda, 0)$ and $m\left(\lambda, \frac{1}{2} \pi\right)$ presented by Chaudhuri and Everitt in [2] since those properties hold equally well for a differential expression $M[f]$ considered on a bounded interval $[a, b)$ with a singular endpoint $b$.

3. Examples. We conclude with an example. Consider inequality (1.1) with

$$
a=0, \quad b=1, \quad p(x)=1, \quad q(x)=3 / 4(1-x)^{2} \quad(x \in[0,1)) .
$$

Then the conditions imposed by Everitt and Giertz [4] are satisfied as is the Dirichlet condition (1.6)(i) at the singular point 1 . Therefore, the differential expression $M[f]$ for this example is strong limit-point at 1 . Also, $\int_{0}^{1} q_{+}=\int_{0}^{1} q$ $=\infty$. Thus all the conditions are satisfied and (1.1) holds for the example described by (3.1).

We write the differential equation (1.2) as

$$
-y^{\prime \prime}+\left(v^{2}-1 / 4\right)(1-x)^{-2} y=\lambda y \quad(0 \leqslant x<1)
$$

where $\nu=1$. Then the Weyl $m$-coefficient $m\left(\lambda, \frac{1}{2} \pi\right)$ is given by

$$
m\left(\lambda, \frac{1}{2} \pi\right)=2 J_{1}(s) /\left(J_{1}(s)+2 s J_{1}^{\prime}(s)\right)
$$

where $s^{2}=\lambda, 0 \leqslant \arg \lambda<2 \pi, 0 \leqslant \arg s<\pi$, and $J_{1}$ is the Bessel function of order 1 of the first kind. (See Titchmarsh [7, §4.8].) We note that $m$ is meromorphic and therefore the spectrum is discrete. Moreover, $\mu_{0}^{1 / 2}$ is the first positive zero of $J_{1}(s)+2 s J_{1}^{\prime}(s)$. The equalizing function (eigenfunction) is then given by

$$
\psi(\gamma)=(1-x)^{1 / 2} J_{1}(s(1-x)) \quad(0 \leqslant x<1) .
$$

\section{REFERENCES}

1. J. S. Bradley and W. N. Everitt, Inequalities associated with regular and singular problems in the calculus of variations, Trans. Amer. Math. Soc. 182 (1973), 303-321. MR 48 \#8943.

2. J. Chaudhuri and W. N. Everitt, On the spectrum of ordinary second-order differential operators, Proc. Roy. Soc. Edinburgh A 68 (1969), 95-119.

3. W. N. Everitt, $A$ note on the Dirichlet condition for second-order differential expressions (to appear).

4. W. N. Everitt and M. Giertz, A Dirichlet type result for ordinary differential operators, Math. Ann. 203 (1973), 119-128. MR 49 \#10.

5. H. Kalf, Remarks on some Dirichlet type results for second-order Sturm-Liouville operators, Math. Ann. 210 (1974), 197-205. 
6. M. A. Naĭmark, Linear differential operators, GITTL, Moscow, 1954; English transl., Part II, Ungar, New York, 1968. MR 16, 702; 41 \# 7485.

7. E. C. Titchmarsh, Eigenfunction expansions associated with second-order differential equations. Part I, 2nd ed., Clarendon Press, Oxford, 1962. MR 31 \#426.

Department of Mathematics, University of Tennessee, Knoxville, Tennessee 37916 (Current address of J. S. Bradley)

Current address (W. N. Everitt): Department of Mathematics, The University of Dundee, Dundee, Scotland 